\title{
Trapping and V3-radial artery graft-V4 bypass for ruptured dissecting aneurysm of the vertebral artery
}

\author{
Tomohiro Inoue, M.D., Akira Tamura, M.D., Ph.D., and Isamu Saito, M.D., Ph.D. \\ Department of Neurosurgery, Fuji Brain Institute and Hospital, Shizuoka, Japan
}

The authors show a surgical technique of trapping/resection of ruptured dominant vertebral artery aneurysm in conjunction with reconstruction of vertebral artery by $\mathrm{V} 3$-radial artery (RA) graft-V4 bypass through suboccipital craniotomy and far lateral approach. Step by step muscle dissection in posterior fossa enable fine exposure of occipital artery for possible OA-PICA bypass and V3 portion of vertebral artery. Extradural drilling of posterior one-third condyle and condylar fossa facilitate exposure of triangular surgical corridor made by medulla, spinal root of 11th nerve and lower cranial nerves, and thus enabling aneurismal resection and RA-V4 anastomosis.

The video can be found here: http://youtu.be/LxsARGdHSVw.

KEY WORDS dissecting aneurysm; vertebral artery; radial artery graft 